\title{
Pulmonary Hyaline Membrane: Late Results of Injury to the Lung Linings
}

\author{
R. A. BARTER*, M. J. BYRNE, and R. F. CARTER \\ From the Departments of Pathology, University of Western Australia and the Adelaide Children's Hospital
}

It has been stated previously that neonatal hyaline membranes are situated in respiratory bronchioles and that they form in situ following necrosis of the lining epithelial cells (Barter, 1959, 1962; Barter and Maddison, 1960). Substances other than products of cellular disintegration may well contribute in the formation of the membranes, but the morphological evidence strongly suggests that the cells normally lining the affected air-spaces undergo degenerative changes and finally necrosis before typical membranes form.

This paper is concerned mainly with the morphology of the later stages of the hyaline membrane and with the results of experiments on rabbits, designed to injure the linings of the distal parts of the respiratory tract. Comparison made between the histological changes in the lungs of newborn infants and lesions resulting from experimental chemical injury provides further evidence that injury to the cellular lining is a primary factor in membrane formation. Lungs from several infants dying during and after the third day and from an infant who survived 102 hours have been especially studied.

\section{Experimental Injury}

The findings reported here formed part of a larger study on experimental lung injury carried out by one of us (M.J.B.). Only details of the work that are relevant to the present observations on human cases will be presented.

Young adult rabbits of mixed breeds and either sex weighing between 1.5 and $3.5 \mathrm{~kg}$. were used to examine the effects of chemical injury to the lung lining. The purpose was to study early and late effects in relation to hyaline membrane disease in terms of epithelial degeneration, necrosis, and regeneration. Two methods were used. First, kerosene was injected intratracheally resulting in damage to the bronchiolar and alveolar

\footnotetext{
Received February 25, 1966.

* Present address: Department of Pathology, King Edward Memorial Hospital for Women, Subiaco, Western Australia.
}

linings and, secondly, mercuric chloride $(\mathrm{N} / 800)$ was. injected intrapleurally resulting in damage to the peripheral alveolar linings. One of the patterns of restorative change following severe injury is pertinent to understanding the late changes observed in human newborn infants. It is the purpose of this description to trace briefly the evolution of the change.

Kerosene series. Young adult rabbits were given a single intratracheal injection of $0.5 \mathrm{ml}$. household kerosene. Test animals and controls were killed by cervical dislocation at selected intervals following injection. At necropsy the trachea was clamped before the thorax was opened, and the lungs were fixed by injection of $10 \%$ buffered formol saline.

The following is a brief description of the microscopical changes. After 2 hours there was a conspicuous cellular exudate located mainly along the surfaces of terminal and respiratory bronchioles. The reaction was well developed at 6 hours in the respiratory bronchioles. Hyaline-like membranes were seen during 8-48 hours but mainly in the period 8-16 hours and were located along the surfaces of respiratory bronchioles (Fig. 1). They varied in thickness from 5 to $60 \mu$ and might be uniformly eosinophilic or show fragments of degenerate nuclear material as slender tapering basophilic masses (Fig. 2).

An inflammatory reaction throughout the lungs increased during 24-48 hours, leading ultimately to dense polymorphonuclear collections. Finally, areas of the lung parenchyma underwent necrosis and abscesses resulted. The surrounding lung showed some compression collapse of the alveoli.

At 48 hours there was a striking new formation of distinctive small epithelial cells in respiratory bronchioles and alveoli (Fig. 3), often demonstrably in the sites of previous hyaline-like membranes.

Mercuric chloride series. Rabbits were injected intrapleurally with N/800 mercuric chloride solution. A single injection was given on the right side and the left lung was used as each animal's own control. Animals were killed by cervical dislocation between 1 hour and 2 months.

One hour after injection there were polymorphonuclear infiltrations in the subpleural tissues immediately preceding fibrinous and cellular exudation in the most 


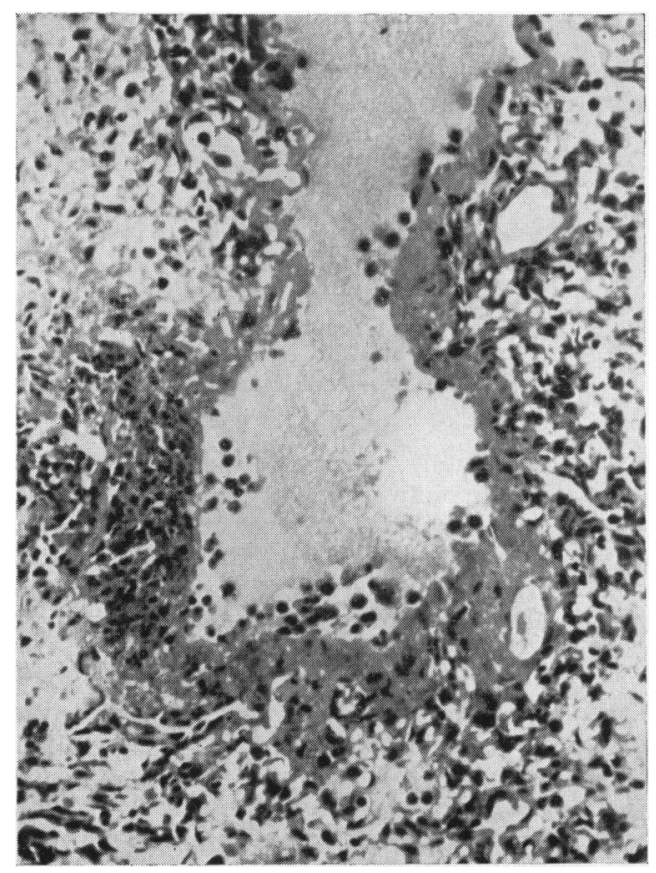

FIG. 1.-Rabbit lung, 48 hours after kerosene injection. Inflammatory reaction and hyaline membrane formation occur along the lining of a respiratory bronchiole. (H. and E. $\times 230$.

peripheral alveoli. At 8 hours the polymorphs reached their maximum numbers and then began to degenerate, with the result that at $\mathbf{3 0}$ hours the exudate was homogeneous and predominantly eosinophilic. The alveolar walls were wide and cellular and contrasted markedly with those in the unaffected parts of the lung.

At 48 hours the peripheral alveoli showed a conspicuous epithelial lining formed of cuboidal and low columnar cells, with darkly staining and relatively large nuclei. Similar cells proliferated on the surfaces of the masses of eosinophilic exudate and also appeared to invade it (Fig. 4). By 72 hours the alveoli showed a complete lining of metaplastic epithelium and most of the exudate had disappeared. The latter apparently occurred as a result of phagocytic activity of the metaplastic cells.

Summarizing the salient findings from both types of chemical injury in rabbits it can be stated that the following sequence of changes occurs in the respiratory bronchioles and alveoli: (1) there is visible evidence of injury to the cellular lining and the formation of an inflammatory exudate; (2) in the case of injury by kerosene, hyaline-like membranes form in respiratory bronchioles after the initial exudative stage; (3) a metaplastic epithelial lining forms deep to the membrane or exudate on the surface of the lamina propria; (4) epithelial cells proliferate over the surfaces of membrane or exudate and subsequently remove either by phago-

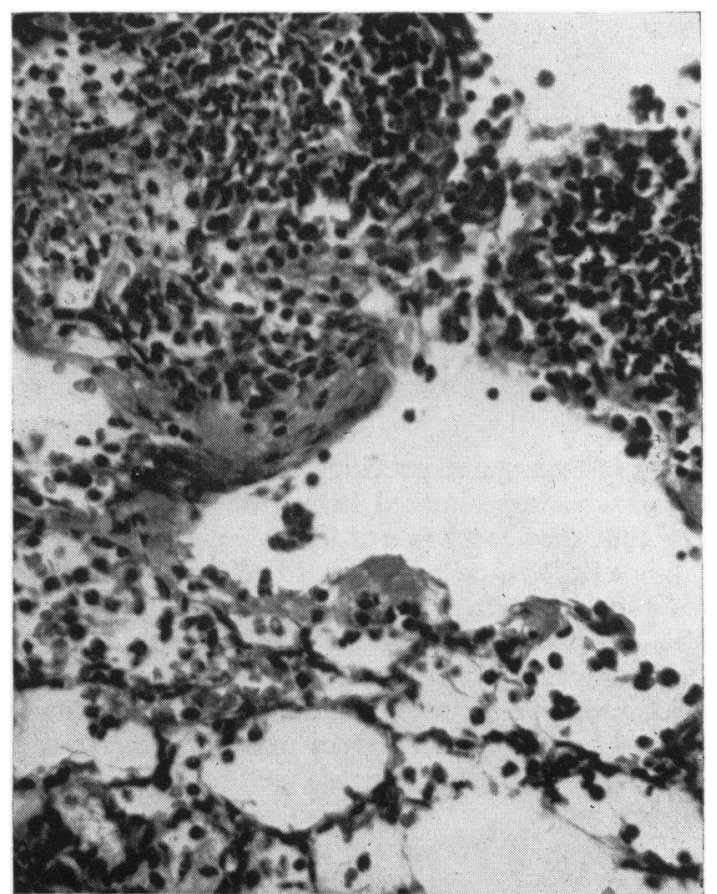

FIG. 2.-Rabbit lung, 8 hours after kerosene injection. $8 \vec{\bullet}$ Hyaline membrane showing tapering masses of basophilic 응 material in the larger mass. (H. and $E . \times 350$.)

cytic activity; (5) the affected air-space is for a time after lined by a conspicuous epithelium, the cells varying from low columnar through cuboidal to pavement.

\section{Late Results in Human Cases}

Hyaline membranes are not seen often in the lungs of infants 4 days old. In fact, they are uncommon in infants who have survived more than 72 hours. To study the morphology of the late stages of the lesion, attention has, therefore, been directed to the lungs showing hyaline membranes from infants surviving more than 48 hours. In these cases there are often conspicuous epithelial cells beneath the apparently friable hyaline membrane (Fig. 5). The cells form against the lamina propria and are clearly responsible for dislodging the membrane into the bronchiolar lumen. Sharp cytoplasmic processes are often seen encircling detached spherical fragments of dense membrane material (Fig. 6). Similar unattached fragments can also be found lying in the bronchiolar lumen.

A somewhat different epithelial reaction was seen in the lungs of an infant described below which survived 102 hours. The striking feature in this 


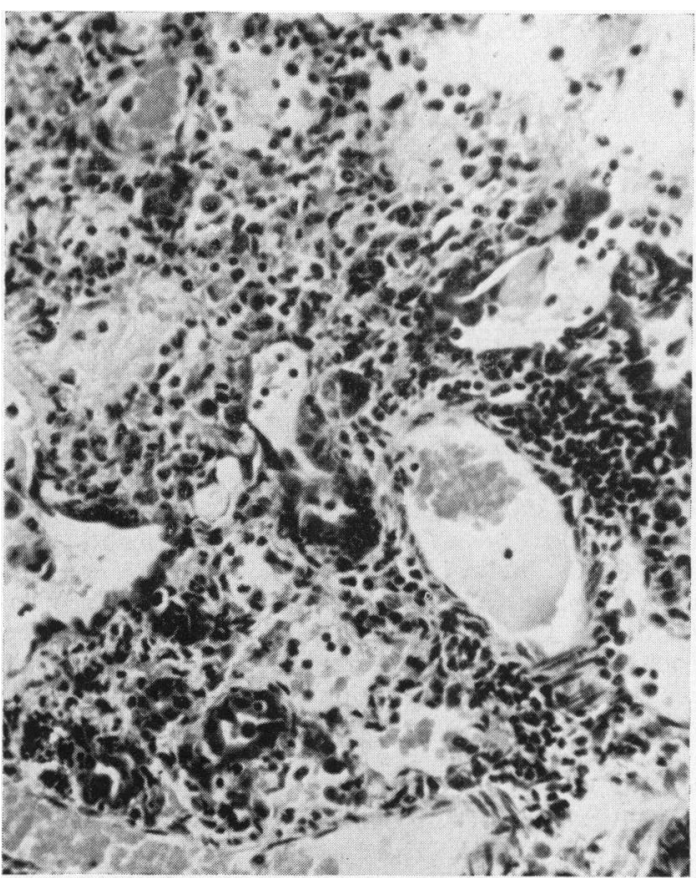

Frg. 3.-Rabbit lung, 48 hours after kerosene injection. Epithelial metaplasia in alveolar and bronchiolar linings. $(H$. and $E . \times 275$.

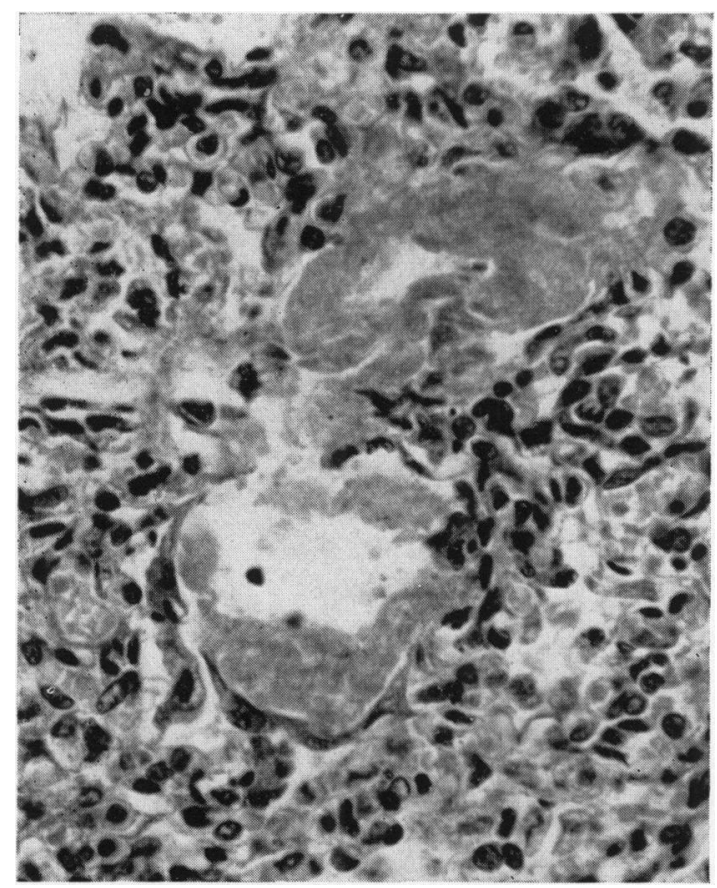

Fig. 5.-Lung from premature infant dying at 64 hours. Metaplastic epithelial cells forming under hyaline membrane. $(H$. and $E . \quad \times 515$.

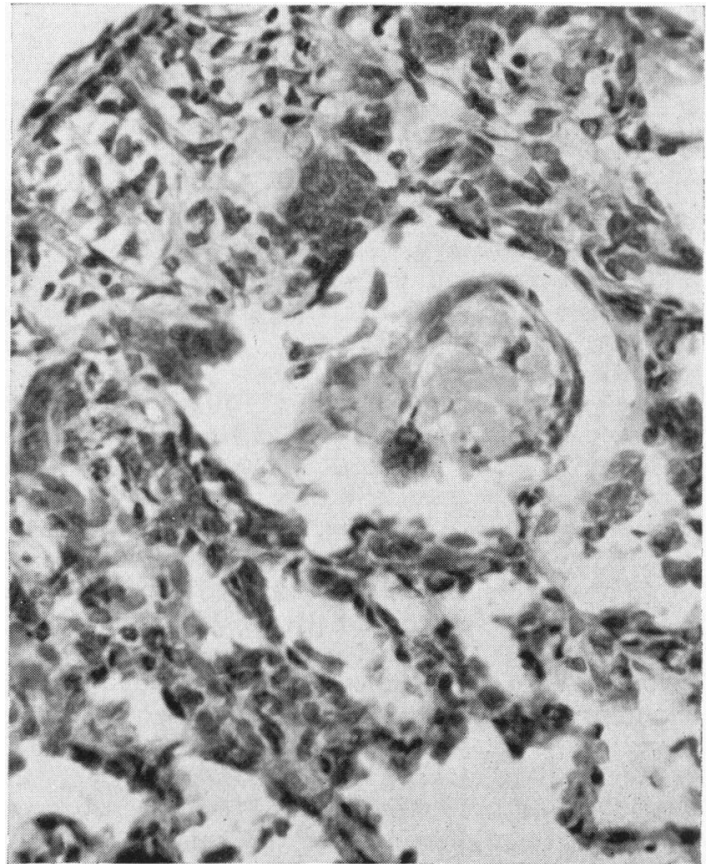

FIG. 4.-Rabbit lung, 72 hours after mercuric chloride injection. Epithelial metaplasia in peripheral alveolar lining. Similar cells surround a proteinaceous exudate which originally filled the lumen. $(H$. and $E . \times 400$. $)$

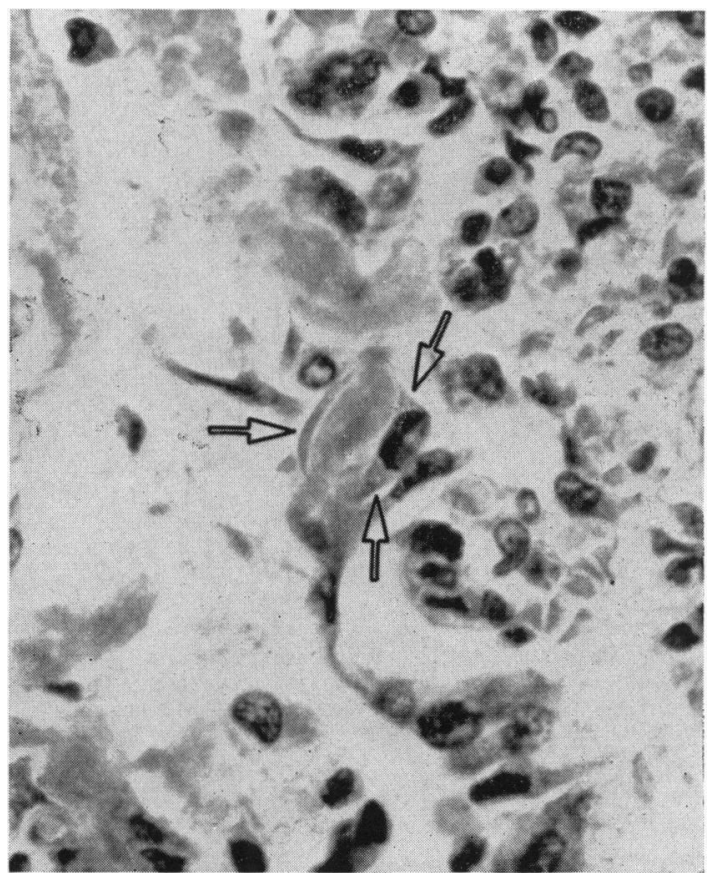

Fig. 6.-Removal of hyaline membrane by epithelial cells. Same case as Fig. 5. Stretched cytoplasmic processes around fragments of membrane. (H. and $E . \times 695$.) 
case was the presence of cells covering the surfaces of the membranes as well as occurring beneath them, as first described.

\section{Case Report}

Clinical summary. A primipara aged 19 years with membranes ruptured for the previous 24 hours was admitted in labour to a maternity hospital in Adelaide. No other details of the pregnancy or of the length of gestation are known.

An oedematous female infant of $970 \mathrm{~g}$. was delivered. She showed marked respiratory distress and an Apgar score of 8 at 8 minutes. Oxygen $(25-30 \%)$ was administered in a humi-crib and i.m. penicillin 50,000 units and streptomycin $25 \mathrm{mg}$. b.i.d. were given.

Respiratory distress with periods of apnoea persisted for 48 hours. Jaundice then developed and ACTH was given. No meconium had been passed and radiograph of the abdomen suggested high intestinal obstruction, probably at the duodenal level. The respiratory condition improved to a degree that additional oxygen was no longer required. The oedema subsided and small quantities of urine were passed. Intermittent gastric suction yielded bile-stained fluid. Surgical treatment was under consideration but the infant's condition deteriorated, and death occurred at the age of 102 hours.

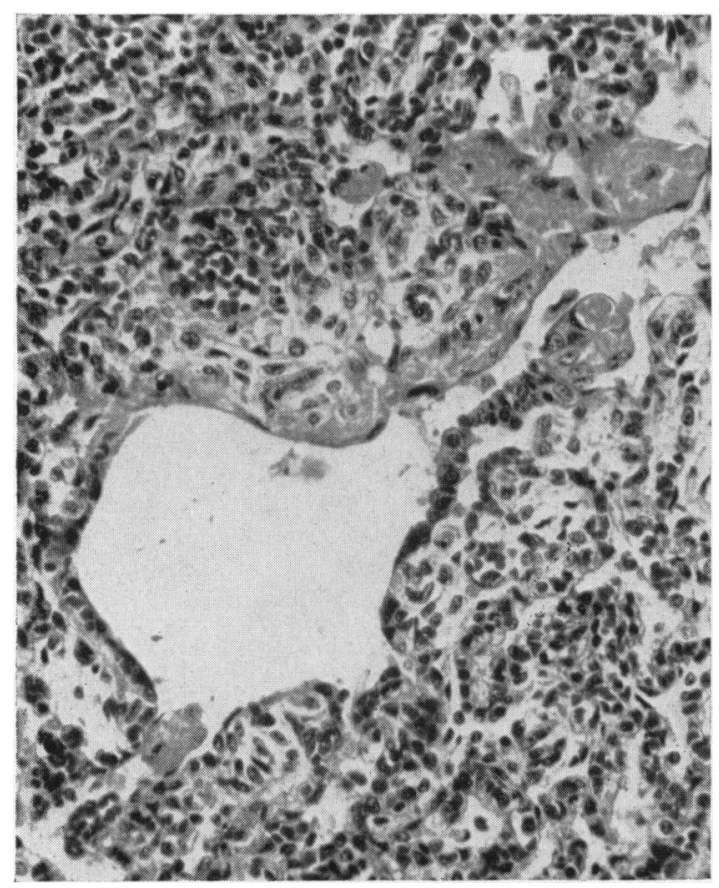

Fig. 7.-Lung from premature infant dying at 102 hours. Hyaline membrane in respiratory bronchiole. Surface covered by epithelium. (H. and $E . \times 240$. $)$
Necropsy findings. The body weighed $975 \mathrm{~g}$. and measured $41 \mathrm{~cm}$. (crown-heel). The duodenum was obstructed in the second part by an annular malformation of the head of the pancreas. The common bile-duct was not identified, but the gall-bladder was much dilated and filled with bile. The small bowel was collapsed and contained dark thick meconium.

The lungs showed slight congestion and oedema with numerous petechial haemorrhages beneath the pleura over the right lower lobe.

Microscopical findings in lungs. The lungs showed only moderate expansion confined to terminal bronchioles and respiratory bronchioles. Hyaline membranes were seen but they were not of usual appearance (Fig. 7), the luminal aspects being covered by epithelial cells. The membrane material sometimes bulged and filled the bronchiolar lumen (Fig. 8). Cells often encircled membrane and were continuous at the periphery with metaplastic lining epithelium. Some parts of the bronchiolar lining were formed only of epithelial cells which were clearly occupying the sites of previous hyaline membrane. These varied from flat cells with greatly extended cytoplasmic margins to small cuboidal cells. The membrane occasionally formed epitheliumcovered cushions at the terminal-respiratory bronchiolar

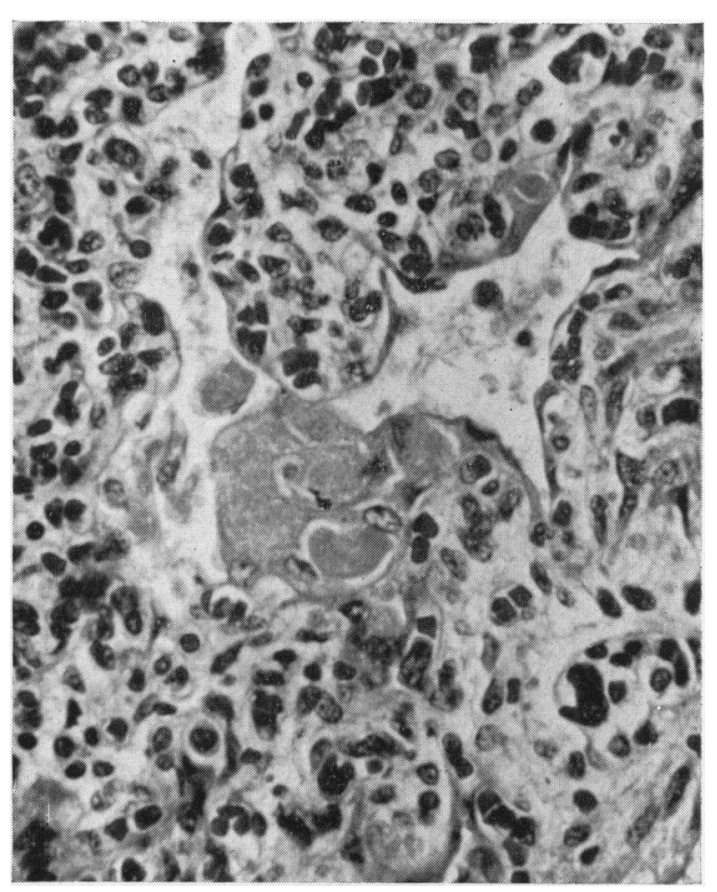

FIG. 8.-Same case as Fig. 7. Disintegrating hyaline membrane and surrounding epithelial cells. Reformation of lining of air-space. (H. and $E . \times 440$. 


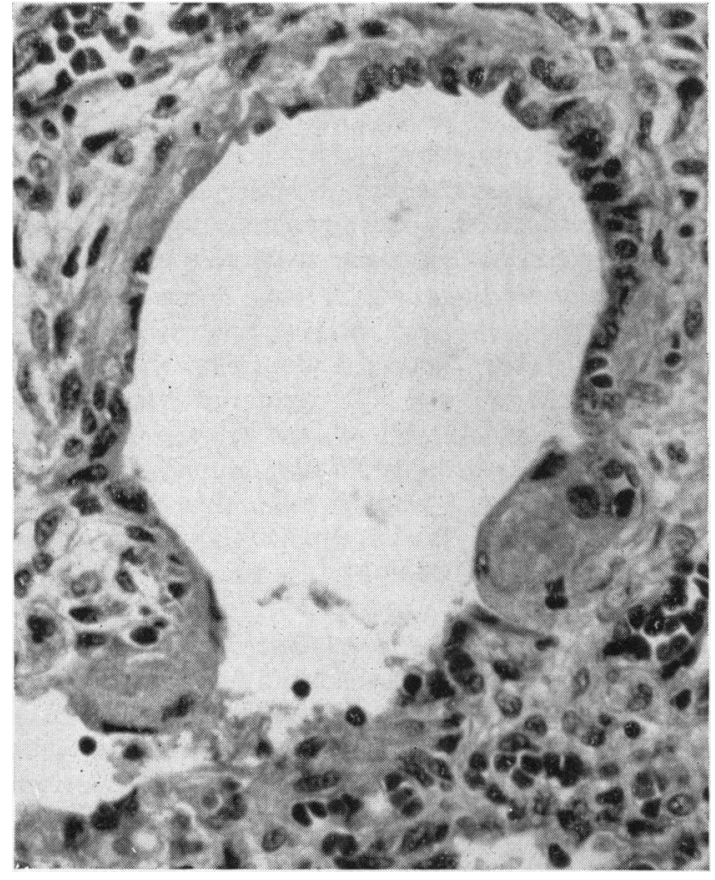

Fig. 9.-Same case as Fig. 7. Epithelialization of surface of hyaline membrane at beginning of respiratory bronchiole. (H. and $E . \times 420$.)

junction (Fig. 9). Masses of material covered by epithelium were often observed in the cross-sections of terminal bronchioles alongside the native epithelial lining (Fig. 10).

One or two of the large masses of membrane material showed faint outlines of degenerate nuclei in their centres. These were the same nuclear remnants that were previously described (Barter and Maddison, 1960) as occurring in the early stages of membrane formation, but were now seen in hyaline material surrounded by epithelial cells which were restoring the respiratory surface.

\section{Discussion}

The changes in the lung in long-standing hyaline membrane disease have not received special attention except for the demonstration by Boss and Craig (1962) that there is evidence of replacement of an epithelial lining in sites of membrane formation. These workers studied a large number of patients who had survived 24 hours or more. They stated that their findings indicated the occurrence of a secondary, regenerative, proliferative response of surviving alveolar cells, which appeared to follow a destructive process associated with the formation of hyaline membranes. They also noted that the newly-proliferated cells might serve as epithelial lining cells; or that they might form macrophages or

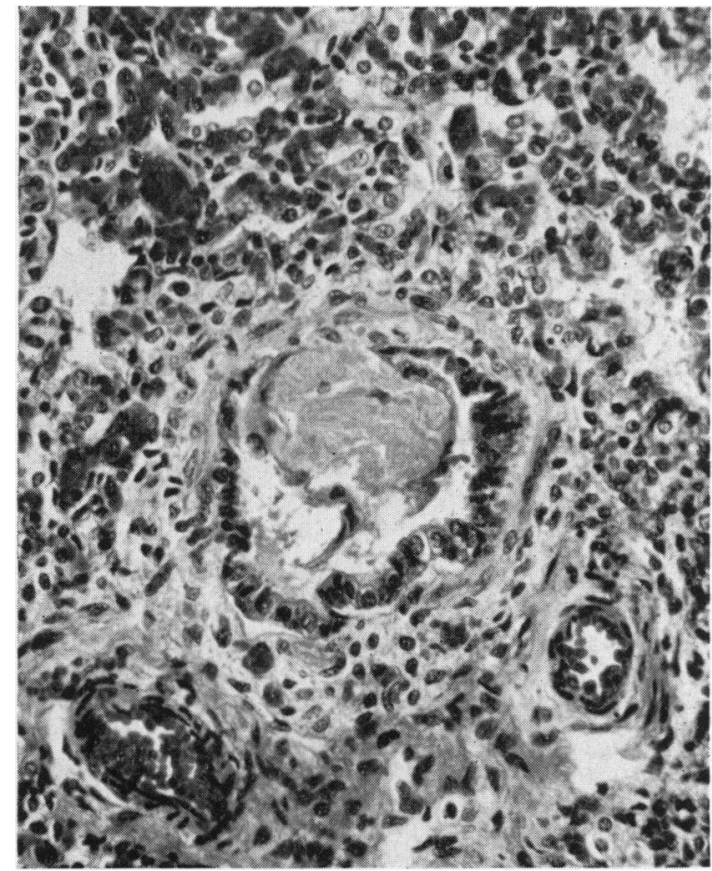

FIG. 10.-Same case as Fig. 7. Hyaline membrane covered by epithelium projecting into distal end of terminal bronchiole. (H. and E. $\times 275$.)

occasionally fibroblasts, the latter giving rise to thickened alveolar walls.

There are no defined criteria for estimating the age of hyaline membranes, and it does not always seem possible to decide whether they formed many hours or immediately before death. As already emphasized, membrane formation can be seen less than 3 hours after birth and can be then clearly related to necrosis of the cells lining respiratory bronchioles, especially when studied in the very immature lung (Barter, 1962). The present cases indicate a late stage characterized by the emergence of a metaplastic epithelium and the removal of the membrane by the phagocytic activity of epithelial cells. It thus seems possible to indicate 5 stages in the evolution of the neonatal pulmonary hyaline membrane: (1) where it is evident that the membranes contain much nuclear debris; (2) where they show a uniform eosinophilic appearance; (3) where a new epithelium has formed between the membrane and the lamina propria or where cells have proliferated over the surface; (4) where the membrane has been removed and the respiratory bronchiole has an abnormal metaplastic epithelial lining; and (5) where the lining has reverted from the conspicuous metaplastic type to the inconspicuous normal 
respiratory bronchiolar epithelium. Time intervals between these stages are not known, and it appears that the changes may proceed at different rates in individual cases.

Considering the foregoing, it might be reasonable to suggest now that from (a) the site of membrane formation, (b) the frequently visible nuclear constituents, and (c) the subsequent reparative processes, there is strong evidence for injury to the epithelial lining in the initial stages. Detailed study of conventionally-stained sections from a large number of cases shows beyond any reasonable doubt that membranes form in the site of a normal lining of epithelial cells which can no longer be discerned once the eosinophilic stage of the membrane formation is reached.

Recent observations (Barter, 1962) suggested a likely approach to studying the pathogenesis of the hyaline membranes by following the effects of chemical injury to the lung lining. Based on the experimental work of others, notably Young (1928, 1930) and Ross (1939), on induced metaplasia in the alveolar lining, it was considered worth while to study the changes that were related to metaplastic transformation following chemical injury.

Acute chemical irritation of the rabbit lung using kerosene leads initially to damage to the linings of respiratory bronchioles and to an almost immediate polymorphonuclear exudation on the damaged surface. In 8 hours there are hyaline-like membranes which are predominantly eosinophilic but contain basophilic nuclear remnants. Somewhat less than 48 hours later an abnormal epithelial lining has formed in place of the membrane. Identical changes occur in some neonatal cases surviving about 48 hours, with an essentially similar process of metaplastic replacement of the normal epithelial lining after clearance of the hyaline material.

Injury caused by solutions of mercuric salts injected into the pleural space leads to the accumulation of a concentrated exudate in the peripheral alveoli. This soon becomes surrounded by phagocytic epithelial cells which are continuous with concurrently developed abnormal cells forming the alveolar lining. A strictly comparable lesion composed of hyaline membrane material and metaplastic epithelial cells is observed in some human newborn infants, and was particularly well seen in the lungs of the infant who survived 102 hours. It is, therefore, suggested, as a result of this morphological similarity, that this pattern of late reaction in human cases might also have followed severe injury to the linings of respiratory bronchioles.

From a consideration of the various stages in the formation and removal of pulmonary hyaline membranes and certain related experimental observations presented here, it now seems reasonable to state precisely that hyaline membranes form in sites usually occupied by respiratory epithelium after injury to that epithelium and its subsequent necrosis. The cause of the necrosis might indeed be more likely to be determined if it were generally conceded that this occurred as an essential phase of the disease.

The new findings which have been presented concern: the cellular reparative processes in wellestablished cases; further evidence for the respiratory bronchiolar site of membrane formation; epithelial phagocytosis of the abnormal lining material; the possibility of the persistence of an abnormal cellular lining in cases that survive the acute disease; and, finally, the likelihood that severe injury to the lung lining itself is the prime cause of hyaline membrane formation.

The actual hyaline membrane is certainly only one of the changes in the affected lung, but it is the visible lesion on which the diagnosis of the disease is made after death and which gives the disease its name. Contrary to the statement by Morison (1963), we believe that necroses in bronchiolar epithelium are primary and also significantsignificant in that they point conclusively to severe injury in the lung.

It is pertinent now to suggest the possibility of integrating the views previously expressed regarding cellular necrosis, with those of Avery and Mead (1959) concerning the loss of surfactant in relation to hyaline membrane formation. We suggest that epithelial damage, probably necrosis, occurs in the alveoli as well as in respiratory bronchioles, and there results in an alteration in the surface properties, which causes pulmonary collapse. The lesion higher in the respiratory lining (hyaline membrane) for one reason or another is the visible degenerative change, in contrast to that in the alveoli which is invisible by light microscopy. Further studies of the alveolar lining, accurately distinguished anatomically, are now required to elucidate the nature of the probable changes in alveoli. It is stressed once more that hyaline membranes can form in the respiratory bronchioles of infants too immature to show alveolar development, and from this it is concluded that an alveolar abnormality is not the cause of the bronchiolar lesion nor, conversely, are hyaline membranes in respiratory bronchioles the cause of the pulmonary collapse. It seems far better to postulate cellular injury at different levels of the respiratory lining from the distal end of terminal bronchioles throughout the respiratory bronchioles to the alveolar sacs.

There is much yet to be discovered concerning the 
initial insult in hyaline membrane disease, but conventional morphological studies may still profitably be made to discern perhaps otherwise unappreciable processes in the evolution of a series of cellular reactions to injury.

\section{Summary}

Stages in the evolution of human pulmonary hyaline membranes have been defined. In particular a late stage characterized by phagocytosis of the membrane and metaplastic reformation of the lining has been stressed. A similar pattern of change has been shown both in the late stages of the disease in human cases and in experimentally induced lung injury. The latter findings seem pertinent in understanding the pathogenesis of the late human lesion and suggest that severe injury may be the initial factor in the naturally occurring disease.

It seems that hyaline membrane in the respiratory bronchioles is merely one facet of a widespread cellular injury in the lung. An injury now postulated in alveoli cannot be detected by the light microscope but may well be manifest as a loss of surface active properties.

Some very prematurely born infants do not have alveolar tissues but may show typical hyaline membrane formation in respiratory bronchioles.

We gratefully acknowledge the permission of Professor G. Maxwell and the honorary staff of the Adelaide Children's Hospital to publish details of one of the cases. Valuable assistance was given by $\mathrm{Mr}$. $\mathrm{H}$. Upenieks in the preparation of the photomicrographs.

\section{REFERENCES}

Avery, M. E., and Mead, J. (1959). Surface properties in relation to atelectasis and hyaline membrane disease. Amer. F. Dis. Child., 97, 517.

Barter, R. A. (1959). The neonatal pulmonary hyaline membrane. Lancet, 2, 160.

(1962). Pulmonary hyaline membrane: further observations on epithelial origin. Arch. Dis. Childh., 37, 314.

$\longrightarrow$, and Maddison, T. G. (1960). The nature of the neonatal pulmonary hyaline membrane. ibid., 35, 460.

Boss, H. H., and Craig, J. M. (1962). Reparative phenomena in lungs of neonates with hyaline membranes. Pediatrics, 29, 890.

Morison, J. E. (1963). Foetal and Neonatal Pathology, p. 205. Butterworth, London.

Ross, I. S. (1939). Pulmonary epithelium and proliferative reactions in the lungs. A study of the cellular response in lungs after intratracheal injection of toxic and nontoxic foreign substances. Arch. Path., 27, 478.

Young, J. S. (1928). Further experiments on the production of hyperplasia in the alveolar epithelium of the lung of the rabbit. f. Path. Bact., 31, 705.

- (1930). Epithelial proliferation in the lung of the rabbit, brought about by intrapleural injection of solutions of electrolytes; a physico-chemical interpretation of the phenomenon. ibid., 33, 363. 